\title{
Defect shape and size discrimination using infrared vibrothermography
}

by A. Mendioroz* ${ }^{*}$ A. Salazar* and R. Celorrio**

*Departamento de Física Aplicada I, Escuela Técnica Superior de Ingeniería, Universidad del País Vasco, Alda. Urquijo s/n, 48013 Bilbao, Spain, arantza.mendioroz@ehu.es, agustin.salazar@ehu.es

**Departamento de Matemática Aplicada, EINA/IUMA, Universidad de Zaragoza, Campus Río Ebro, Edificio

Torres Quevedo, 50018 Zaragoza, Spain, celorrio@unizar.es

\begin{abstract}
Vibrothermography has demonstrated its ability for the detection of defects that are elusive for other nondestructive techniques in a wide range of materials. However, its capability for characterizing such defects is still under investigation. In this work we analyze both theoretically and experimentally the ability of lock-in vibrothermography to distinguish between different defect shapes and sizes. We compare calculated surface thermograms computed for buried heat sources of different shapes, sizes and depths. Experimental data obtained with calibrated heat sources confirm the predictions of the calculations.
\end{abstract}

\section{Introduction}

Lock-in Infrared thermography by modulated ultrasound excitation was first introduced in the late seventies [1]. It has recently attracted much attention because of its ability for finding defects which cannot be detected by other nondestructive techniques [2,3]. However its development is burdened by certain experimental issues (reproducibility troubles or strong signal dependence on experimental configuration) and by difficulties in understanding the signal generation mechanisms. In addition, although some work has been done intended to test its potential to characterize simple shaped defects [4], its capabilities to distinguish between different defect shapes have not been studied yet. In this work we analyze theoretically the ability of lock-in vibrothermography to distinguish between different hidden defect shapes. Besides, we test the predictions of our calculations by comparing them to lock-in vibrothermography data obtained from samples containing calibrated buried defects.

\section{Theory and simulations}

We consider defects of arbitrary shape located in planes forming any angle with the surface: from parallel (representing delaminations) to perpendicular (representing vertical cracks). First we solve the direct problem: we calculate the surface temperature amplitude and phase corresponding a particular shape by adding the contributions to the surface temperature $(z=0)$ of point-like heat sources modulated at frequency $f$ and located at coordinates $\left(x_{o}, y_{o}, z_{o}\right)$, as shown in Fig. 1:

$$
T(x, y, 0)=T_{o} \frac{e^{-q \sqrt{\left(x-x_{0}\right)^{2}+\left(y-y_{o}\right)^{2}+z_{o}^{2}}}}{\sqrt{\left(x-x_{0}\right)^{2}+\left(y-y_{0}\right)^{2}+z_{0}^{2}}}
$$

By numerically integrating the expressions corresponding to each particular heat source, we calculate the surface temperature amplitude and phase at the desired modulation frequency.

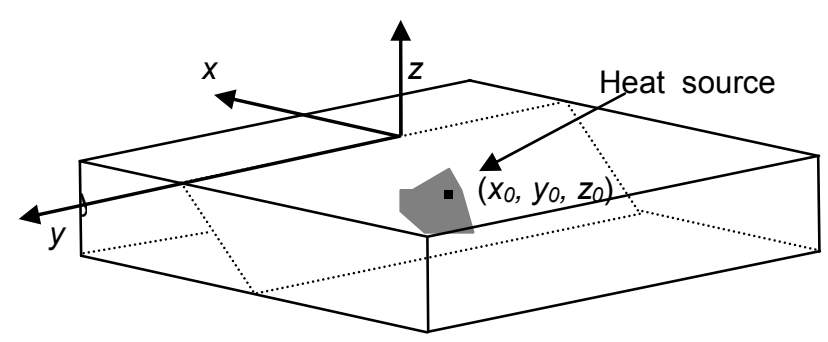

Fig. 1. Diagram of an inclined crack

\section{Experiments and discussion}

The measurements were performed using commercial lock-in vibrothermography equipment (UTvis from EDEVIS). The surface temperature data were collected with an infrared camera (Jade J550M from CEDIP), synchronized with the amplitude varying input signal. We have prepared samples simulating the presence of a heat source by attaching two pieces of AISI 304 stainless steel against each other through a flat surface of dimensions $1.5 \mathrm{~cm}$ $\times 1.5 \mathrm{~cm}$. In order to better localize the heat sources in an easy way, we have introduced between these two pieces a 
thin sheet of copper, whose shape and size is varied in different experiments. The dimensions of the copper sheet are at least one order of magnitude smaller than those of the flat surface. As an example in Fig. 2a we show a micrograph of the plane containing the heat source.

In Fig. $2 \mathrm{~b}$ we show the surface temperature phase thermogram corresponding to the sample shown in Fig. $2 a$, obtained at a modulation frequency of $3.4 \mathrm{~Hz}$. For each defect configuration, we have compared the experimental data to surface temperature amplitude and phase calculations computed with heat source parameters corresponding to the experimental samples. In fig. $2 \mathrm{c}$ we show in symbols the experimental phase corresponding to profiles 1 and 2 of Fig. $2 \mathrm{~b}$, together with the calculated phase data (solid line).
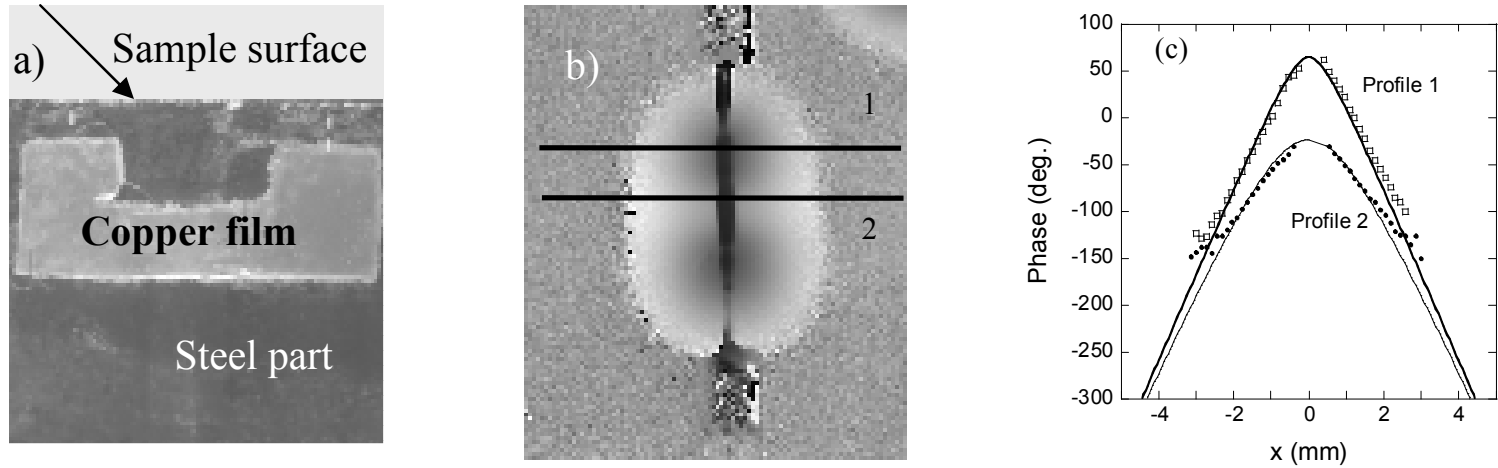

Fig. 2. (a) Cross-section of the sample along the vertical crack plane simulated by a copper film. (b) Corresponding experimental surface temperature phase map obtained at $3.4 \mathrm{~Hz}$. (c) Experimental (symbols) and computed (solid lines) phase profiles corresponding to the lines depicted in (b).

As can be observed, the agreement between theory and experiment is good. However, preliminary theoretical calculations we have performed for heat sources perpendicular to the surface of similar size but different shapes indicate that only when the upper contours (closer to the surface) of the heat sources are different, the resulting surface thermograms are distinguishable. This conclusion is consistent to the damped nature of thermal waves. Additional calculations are currently being performed to determine the conditions in which different heat source shapes can be distinguished in a lock-in vibrothermography experiment.

\section{Summary and Conclusions}

We have calculated the surface temperature amplitude and phase generated by intensity modulated buried heat sources of arbitrary shape, size and orientation. The results indicate that the most important effect for the thermograms to be distinguishable is the upper contour of vertical cracks. Vibrothermography data obtained with samples containing calibrated heat sources are in good agreement with the theoretical calculations.

\section{ACKNOWLEDGMENTS}

This work has been supported by the Basque Government (S-PE11UN024) and by the Ministerio de Educación y Ciencia (MAT2008-01454).

\section{REFERENCES}

[1] Carlomagno, G. M., Berardi P. G., "Unsteady thermotopography in non-destructive testing", $3^{\text {rd }}$ Biannual Exchange Proceeding, St. Louise, (USA), pp 33-39, 1976.

[2] Gleiter A., Spieß $\beta$ berger C., Busse G., "Lockin-thermography with optical or ultrasound excitation" The $10^{\text {th }}$ International Conference of the Slovenian Society for Non-Destructive Testing Proceedings, Ljubljana (Slovenia) pp. 447-454 (2009).

[3] Piau J.-M., Bendada A., Maldague X., Legoux j.-g., "Nondestructive testing of open microscopic cracks in plasma-spayed-coatings using ultrasound excited vibrothermography", Nondestructive Evaluation and Testing, vol. 23, pp. 109-120 (2008)

[4] Mendioroz A., Apiñaniz, E., Salazar A., Venegas P., Sáez de Ocáriz I., "Quantitative study of buried heat sources by lock-in vibrothermography: an approach to crack characterization", Journal of Physics D: Applied Physics, vol. 42, pp 055502-1/055502-9 (2009). 